\title{
Low-energy signatures of charge and spin fluctuations in Raman and optical spectra of the cuprates
}

\author{
S. Caprara, C. Di Castro, T. Enss, and M. Grilli \\ SMC - Istituto Nazionale per la Fisica della Materia, and Dipartimento di Fisica, Università "Sapienza", Roma, Italy
}

\begin{abstract}
We calculate the optical and Raman response within a phenomenological model of fermion quasiparticles coupled to nearly critical collective modes. We find that, whereas critical scaling properties might be masked in optical spectra due to charge conservation, distinct critical signatures of charge and spin fluctuations can be detected in Raman spectra exploiting specific symmetry properties. We compare our results with recent experiments on the cuprates.
\end{abstract}

Key words: Stripes; Quantum critical point; spectroscopy PACS: 74.72.-h, 78.30.-j, 71.45.Lr, 74.20.Mn

\section{Introduction}

It has been proposed that the anomalous physical properties of the cuprates arise from the proximity to a quantum critical point (QCP) located near optimal doping $[1,2,3,4]$. The onset of the ordered phase, however, can be prevented by the low dimensionality of the $\mathrm{CuO}_{2}$ layers, by disorder, and/or by the occurrence of pairing, thereby making the order that should prevail in the low-temperature underdoped region rather elusive. Besides the possibility of unusual ordered states [2,5], experiments indicate that charge ordering $(\mathrm{CO})$, possibly in the form of stripe or checkerboard textures, is indeed present in underdoped superconducting cuprates. A survey is provided in Refs. $[1,6]$.

Based on the experimental evidence and on theoretical results within models for strongly correlated electrons, a scenario was proposed [4], where quantum criticality near optimal doping and $\mathrm{CO}$ in the underdoped region merge in a unified scenario. Furthermore, due to the closeness to an antiferromagnetic (AF) Mott-insulating phase at very low doping, also the presence of substantial AF spin fluc- tuations could markedly affect the physical properties of these systems [7]. The roles of AF and charge fluctuations are not necessarily exclusive: The former could rather be emphasized by $\mathrm{CO}$, and be "enslaved" in the charge-poor regions produced by the modulation of the charge profile, thus extending their relevance to doping levels substantially higher than the AF onset.

Within a model of strongly interacting electrons coupled to a dispersionless phonon [4], for realistic model parameters, the $\mathrm{CO}$ instability first occurs along the $(1,0)$ or $(0,1)$ directions (i.e., along the $\mathrm{Cu}-\mathrm{O}$ bonds) with typical wavelength of a few lattice spacings. Accordingly, near the QCP the fermion quasiparticles (QP) are coupled to low-energy CO collective modes (CM), and are strongly affected near the "hot" spots at the Fermi surface, which are mutually connected by th critical wavevector $\mathbf{q}_{c}$ and reside near the points $( \pm \pi, 0)$ and $(0, \pm \pi)$ of the Brillouin zone. A similar phenomenology is found in the case of AF spin fluctuations, although with a different characteristic wavevector, $\mathbf{q}_{s} \approx(\pi, \pi)$. The effects of the exchange of nearly critical CM have been extensively studied within phenomenological 
models with QP-CM coupling. In this way, specific features in ARPES (e.g., the QP self-energy [8], the kink [9] and the isotopic dependence of the dispersions [10]), and anomalous isotopic effects [11] have been coherently explained within the CO-CM scenario.

In this paper, we explore the optical and Raman signatures of the $\mathrm{CO}$ and of the (enslaved) AF spin fluctuations, in particular (a) the possibility to detect spectroscopic features displaying scaling properties as fingerprints of quantum critical CM and (b) how the symmetry properties of the Raman selection rules determine the relevance of the spin and/or charge $\mathrm{CM}$ at different energy scales.

\section{Critical collective modes in optical spectra}

Near the CO instability (i.e., near the QCP at optimal doping and in the underdoped, on the verge of $\mathrm{CO}$ ), the $\mathrm{CO} \mathrm{CM}$ have a small mass and provide a "cheap" reservoir of excitations, which can easily affect the various spectroscopic probes.

Recently, we performed a microscopic calculation of the optical conductivity $\sigma(\omega)$ [12], based on a conserving approximation of the current-current response, which is crucial to correctly describe optical absorption. It was important to realize that for a parabolic electron dispersion and in the absence of quenched impurities (clean case), the CM cannot be purely electronic and must involve, e.g., phonons, otherwise the conservation of electron momentum would entail a vanishing $\sigma(\omega)$ at $\omega>0$. Phonons modify the purely electronic relaxational form of the $\mathrm{CO} \mathrm{CM}$ propagator, yielding an effective interaction

$D\left(\mathbf{q}, \omega_{n}\right)=-\frac{g^{2}}{\nu\left(\mathbf{q}-\mathbf{q}_{c}\right)^{2}+\left|\omega_{n}\right|+\omega_{n}^{2} / \bar{\Omega}+m}$

among the QP. Here, $\omega_{n}$ are boson Matsubara frequencies, $g$ is the QP-CM coupling, $\nu$ is a fermion scale, the high-frequency cutoff $\bar{\Omega}$ relates fermion and phonon scales [12] and encodes the dynamical nature of the phonons, and $m \propto \xi^{-2}$ is the $\mathrm{CO} \mathrm{CM}$ mass, proportional to the inverse square correlation length measuring the distance to the $\mathrm{CO}$ transition. A similar effective interaction describes also the $\mathrm{AF}$ $\mathrm{CM}$, with a proper redefinition of the parameters, and yields very similar results for $\sigma(\omega)$. For $\bar{\Omega}<\infty$, a finite response was found, with various regimes for $\sigma(\omega)[12]$. At low temperature $T$, the near absence of dissipation for $\omega<\bar{\Omega}$ strongly suppresses $\sigma(\omega)$, yielding a peak which is fixed at $\omega \sim \bar{\Omega}$ and does

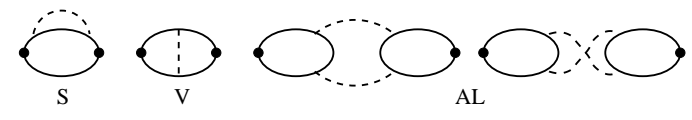

Fig. 1. Diagrams for the CM corrections to the response function. The full dots represent the current vertices in the case of optical conductivity or the Raman vertices. The solid lines represent the QP propagator (with or without impurity scattering) and the dashed lines represent the interaction mediated by CO or AF CM. Self-energy, vertex, and Aslamazov-Larkin-like contributions are labeled by S, V, and AL, respectively.

not scale with $m$ when $m<\bar{\Omega}$. On the other hand, if $m>\bar{\Omega}$, a peak related to the excitation of $\mathrm{CO}$ $\mathrm{CM}$ appears at $\omega \sim m$. At higher $T$, the thermal excitation of the diffusive CM rapidly fills the lowfrequency spectrum, embedding the finite-frequency peaks into a broader peak at $\omega=0$. Therefore, the scaling behavior may be masked by non-universal effects, and we concluded that the lack of scaling peaks in optical spectra cannot be taken as evidence against quantum criticality.

Here, we address the question whether and how the presence of impurities (dirty case) modifies the above conclusions. We determine the conditions for a finite-frequency peak to be visible and display scaling via its mass-dependent position, when an impurity scattering rate $\Gamma$ broadens the QP propagator in Fig. 1. In these diagrams the impurity vertex corrections cancel out, while, owing to the finite $\mathbf{q}_{c}$, no diffusive impurity ladders can be inserted. We assume a CM mass $m \propto T$, as appropriate for the quantumcritical regime, and calculate the corrections $\delta \sigma(\omega)$ arising from the current-current diagrams of Fig. 1 . We first discuss the purely perturbative result, and then introduce a suitable resummation scheme to avoid the shortcomings of perturbation theory.

Impurities enlarge the free-electron $\delta(\omega)$ response into a Drude peak of width $\Gamma$. If $\Gamma$ is of the order of (or even larger than) $m$ and/or $\bar{\Omega}$, this Drude term embeds the peaks due to $\mathrm{CM}$ scattering and makes the critical behavior of the fluctuations difficult to detect. Therefore, a first conclusion is that the observation of peaks due to CM is only possible when disorder is sufficiently weak. To further explore the effects of critical CM in optics we accordingly introduce a rather small disorder scattering rate $\Gamma<$ $m, \bar{\Omega}$. Disorder produces a finite negative part in the CM perturbative correction, $\delta \sigma(\omega)$, at low $\omega$. This is naturally so, because the conservation of the optical weight implies that $\delta \sigma$ has zero total weight, $\int d \omega \delta \sigma(\omega)=0$. Therefore, when the dirty QP are dressed by $\mathrm{CO} \mathrm{CM}$, the spectral weight added at 
frequencies $\omega \gtrsim \Gamma$ has to be subtracted at $\omega \lesssim \Gamma$. This feature was already present in the clean case, where, however, the negative part was $\delta(\omega)$-like and did not affect the spectra at finite $\omega[12]$.

In the presence of impurities, the negative part of $\delta \sigma$ may prevail despite the Drude peak even for small or moderate QP-CM coupling $g$, yielding an unphysical negative absorption at low $\omega$. This artifact can be cured by an appropriate resummation scheme, which we propose here. Specifically, we consider a memory function [13], which treats on equal footing the QP scattering due to impurities and to nearly critical CM, $M(\omega)=i \Gamma-\omega \chi_{C M}^{\text {clean }}(\omega) / W$, where $\chi_{C M}^{\text {clean }}$ is the clean $\mathrm{CM}$ correction to the current-current response (see Fig. 1) and $W$ is the free QP optical weight. The corresponding resummed response is

$\chi_{j j}(\omega)=\frac{W \omega}{\omega+M(\omega)}$,

which reproduces the perturbative result at high $\omega$, where $\chi_{C M}^{\text {clean }} / W \ll 1$. In the absence of QP-CM coupling $\chi_{C M}^{\text {clean }}=0$ and we recover the bare Drude response $\chi_{D}=W \omega /(\omega+i \Gamma)$. Although our resummation scheme is no longer conserving, it does not spoil the previous conclusions on the conditions for optical peaks scaling with $m$. Figs. $2(\mathrm{a})$ and (b) report the results for the resummed $\sigma(\omega)=-\operatorname{Im} \chi_{j j}(\omega) / \omega$ for the cases $m>\bar{\Omega}$ and $m<\bar{\Omega}$, respectively.

Our analysis shows that, in the presence of a small amount of impurities, the spectra broaden, but the generic feature persists, and the absence of a $T$ dependence in the peak position [see Fig. 2(b)] cannot be used to infer the absence of critical CM (with quantum-critical mass $m \propto T$ ). The CM become fully visible in optical spectra only at low $T$ and when the CM mass is larger than a typical nonuniversal dissipation scale $\bar{\Omega}$ [see Fig. 2(a)]. Note that the displacement of weight from $\omega \lesssim \Gamma$ to higher frequencies, produced by $\delta \sigma$, can result in a narrowing of the Drude peak. In the analysis of experiments, this could lead to an erroneous overestimate of the QP lifetime. The determination of the precise amount of narrowing and of the extent to which the negative part of $\delta \sigma$ could "carve" a dip in the spectrum, thereby producing a finite-frequency peak despite the rapid thermal filling mentioned above, requires a reliable conserving resummation scheme to treat the low-frequency absorption, which is presently not available, due to the lack of a small expansion parameter.
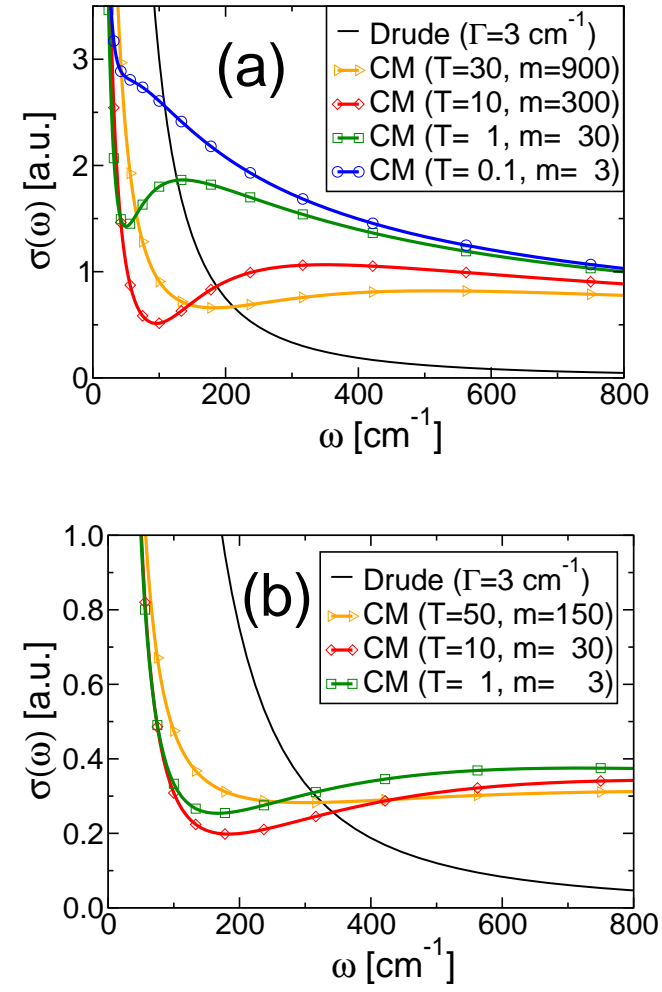

Fig. 2. Optical conductivity for a system of QP coupled to $\mathrm{CO} \mathrm{CM}$ modes in the presence of finite impurity scattering rate $\Gamma=3 \mathrm{~cm}^{-1}$, at various temperatures and CM masses, for (a) $\bar{\Omega}=30 \mathrm{~cm}^{-1}$, and (b) $\bar{\Omega}=330 \mathrm{~cm}^{-1}$.

In summary, within our approach a sufficiently strong disorder may effectively wash out the effects of CM, thereby hiding criticality. When disorder is weak enough to leave the peaks at low $T$ visible, impurities do not qualitatively modify the findings of the clean case: The key role in the non-universal behavior of $\sigma(\omega)$ is played by charge conservation in the presence of an extrinsic (e.g., of phonon origin) energy scale for dissipation $\bar{\Omega}$. The value of the critical scale $m$ in comparison to $\bar{\Omega}$ then determines the presence or the absence of a critical $T$ dependence of the optical features. In cuprates we estimate $\bar{\Omega} \sim$ $100 \mathrm{~cm}^{-1}[12]$.

\section{Critical collective modes in Raman spectra}

The natural question arises on the role of dissipation on the behavior of other spectral quantities, which are not "protected" by charge conservation. Within this respect, Raman spectroscopy provides a nice playground with the additional advantage of a momentum-dependent filtering of the spectra. A 
key difference with respect to the optical conductivity is that, properly adjusting the polarization of the incoming and outgoing photons, the Raman response involves form factors selecting specific regions of the QP Brillouin zone [16]. This helps to distinguish nearly critical AF and $\mathrm{CO}$ fluctuations in Raman spectra, which would look very similar in optical spectra [14].

To compare Raman and optical spectra, we consider here the contribution to the Raman response due to AF and CO CM. Since we have shown that impurity scattering should be weak enough not to hide the effects of $\mathrm{CM}$ in optics, we henceforth focus on the role of $\mathrm{CM}$ and neglect impurities. Specifically, we consider the clean Raman response $\operatorname{Im} \chi$ in the $B_{1 g}$ and $B_{2 g}$ channels, where photons couple to $\mathrm{QP}$ via the vertices $\gamma_{B_{1 g}}=\cos k_{x}-\cos k_{y}$ and $\gamma_{B_{2 g}}=$ $\sin k_{x} \sin k_{y}$. While $\gamma_{B_{1 g}}$ is large (and therefore selects excitations) in the antinodal regions around the $( \pm \pi, 0)$ and $(0, \pm \pi)$ points of the Brillouin zone and changes sign in the nodal regions $k_{x}= \pm k_{y}$, $\gamma_{B_{2 g}}$ is large around the nodes and changes sign in the antinodal regions. These symmetry properties induce leading-order cancellations between the Raman response diagrams depending on the momentum exchanged by the CM. For instance, we find that the $\mathrm{S}$ and $\mathrm{V}$ diagrams (see Fig. 1) cancel each other in the $B_{1 g}$ channel, when typical momenta $\mathbf{q}_{c}$ of $\mathrm{CO}$ excitations are exchanged, because $\gamma_{B_{1 g}}$ has the same value at the two hot spots connected by $\mathbf{q}_{c}$, and the $\mathrm{S}$ and $\mathrm{V}$ diagrams have intrinsically opposite sign 1 . In this case, the only relevant contribution arises from the AL diagrams, which were considered in Ref. [15], to explain the Raman absorption anomalies observed in $\mathrm{La}_{2-x} \mathrm{Sr}_{x} \mathrm{CuO}_{4}$ (LSCO) with $x=0.02,0.10$ [18]. On the other hand, AF $\mathrm{CM}$ with typical wavevector $\mathbf{q}_{s} \approx(\pi, \pi)$ give qualitatively wrong results. In Ref. [15], a diffusive CM propagator was considered, mediating an effective interaction similar to that in Eq. (1), but with a different high-frequency behavior. Here, having in mind a comparison with the results on $\sigma(\omega)$, obtained using Eq. (1), we reproduce the analysis of Ref. [15] using the high-frequency cutoff $\bar{\Omega}$. As it can be seen in Fig. 3(a), the experimental data are well reproduced setting $\bar{\Omega}=200 \mathrm{~cm}^{-1}$ and using $m$ as the only fitting parameter, once the overall intensity is set by the curve at the highest tempera-

\footnotetext{
1 This cancellation of $\mathrm{S}$ and $\mathrm{V}$ diagrams is reminiscent of the loop cancellation occurring in the density-density response of systems with strong forward scattering [17].
}
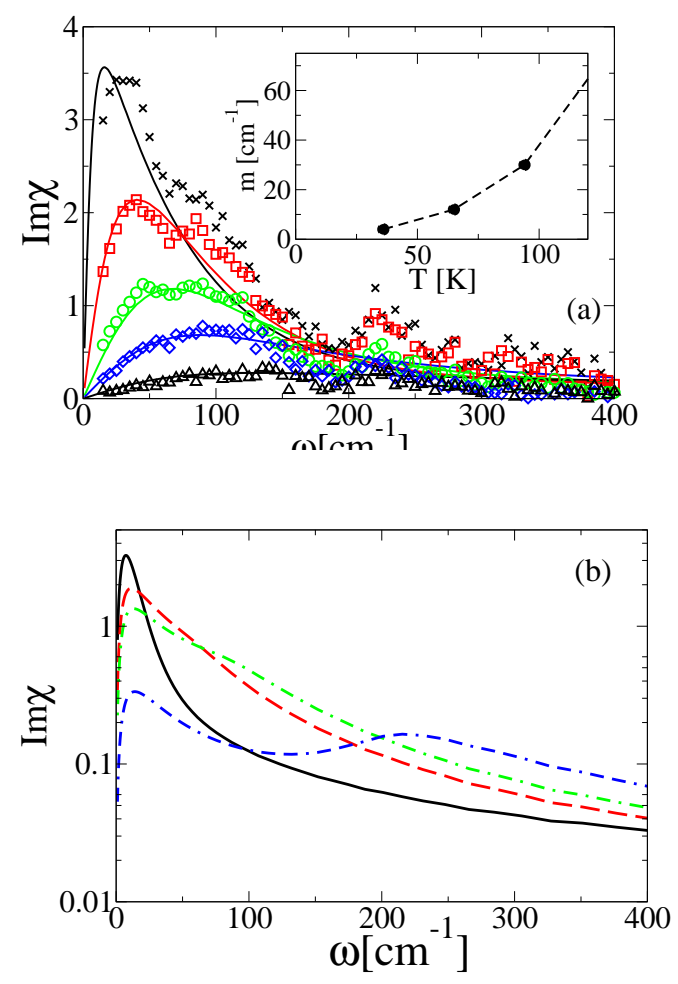

Fig. 3. (a) Raman absorption in the $B_{1 g}$ channel for a clean system of QP coupled to $\mathrm{CO} C \mathrm{CM}$ at temperatures (from top to bottom) $T=36,65,94,124,188 \mathrm{~K}$. The mass $m(T)$ needed to fit the data is reported in the inset. A dissipation scale $\bar{\Omega}=200 \mathrm{~cm}^{-1}$ is used. (b) Same as in (a) with $T=36,94,124,188 \mathrm{~K}$, but with a smaller dissipation scale, $\bar{\Omega}=20 \mathrm{~cm}^{-1}$.

ture. Again, $m(T)$ behaves as expected for a system crossing over from the quantum critical region to the low- $T$ nearly ordered phase. Clearly, due to the inherently non-conserving character of Raman excitations, the scaling behavior of the peak is visible also when $m<\bar{\Omega}$. For the sake of completeness, we also discuss the case $m>\bar{\Omega}$, although it does not apply to LSCO. In Fig. 3(b) we report the $B_{1 g}$ Raman spectra (again coming from the AL diagrams only, for symmetry reasons) for the same masses as in Fig. 3(a), but a smaller $\bar{\Omega}=20 \mathrm{~cm}^{-1}$. Here, a narrow peak appears at a frequency $\omega \sim \bar{\Omega}$, above which the CM changes from diffusive to propagating [see Eq. (1)]. The mass energy scale $m$ is still visible as a shoulder (for low and intermediate $T$ ) or as a broad peak (at the largest temperature $T=188 \mathrm{~K}$ ).

\section{Symmetry properties of Raman spectra}

As mentioned in the previous section, the specific symmetry of Raman vertices was exploited in Ref. 


\begin{tabular}{|c|c|c|}
\hline & $B_{1 g}$ & $B_{2 g}$ \\
\hline $\mathrm{CO} \mathrm{CM}$ & $A L \neq 0, \quad S+V \approx 0$ & $A L \approx 0, \quad S+V \neq 0$ \\
\hline $\mathrm{AF} \mathrm{CM}$ & $A L \approx 0, \quad S+V \neq 0$ & $A L \approx 0, \quad S+V \approx 0$ \\
\hline
\end{tabular}

Leading corrections to the Raman absorption due to the processes represented by the diagrams of Fig. 1, in the $B_{1 g}$ and $B_{2 g}$ symmetries.

[15] to explain the anomalies observed in LSCO [18]. In this section, we focus on those features of Raman spectra which can be discussed based only on symmetry considerations. These provide rather robust arguments on the relevance or irrelevance of the leading contributions from $\mathrm{CO}$ or $\mathrm{AF} \mathrm{CM}$ in the various channels when the CM are nearly critical and strongly peaked at their characteristic wavevectors. On the other hand, far away from criticality, when $m$ is large and the CM mediate smooth and weak interactions, the symmetry arguments are of little use because the $\mathrm{CM}$ correction to the response is small and substantial contributions come from regions of the Fermi surface away from the hot spots.

By direct inspection of the symmetry properties of the QP loops entering the AL diagrams (see Fig. 1 ), one realizes that the leading contributions of $\mathrm{AF}$ CM with $\mathbf{q}_{s}=(\pi, \pi)$ are non-zero only in the $\gamma_{A_{1 g}}=$ $\cos \left(k_{x}\right)+\cos \left(k_{y}\right)$ symmetry [19]. In the $B_{1 g}$ channel, a leading contribution to Raman absorption from the AL diagrams can only arise if the CO CM have a characteristic wavevector $\mathbf{q}_{c}$ in the $(1,0)$ or $(0,1)$ directions [15]. As far as the S and V diagrams are concerned, as anticipated in the previous section, the leading contributions in the $B_{1 g}$ symmetry cancel when the CO CM is considered. In the $B_{2 g}$ symmetry, instead, the $\mathrm{S}$ and $\mathrm{V}$ diagrams sum up (since now the Raman vertex has opposite sign in the two hot spots connected by $\mathbf{q}_{c}$, avoiding loop cancellation) and provide a finite leading contribution. The opposite situation is found when the $\mathrm{CM}$ wavevector is $\mathbf{q}_{s} \approx(\pi, \pi)$, typical of AF CM near the AF instability: due to the symmetry of the form factors, the $\mathrm{S}$ and V diagrams sum up in the $B_{1 g}$ channel, while they cancel at leading order in the $B_{2 g}$ channel. The situation is summarized in Table 1 .

Since the leading $\mathrm{S}+\mathrm{V}$ contributions diverge as $\omega \rightarrow 0$, a resummation is required to obtain a finite Raman response. We adopt the same memoryfunction scheme of Eq. (2) (here we consider the clean case $\Gamma=0)$. We calculate the corrections to Raman absorption due to both AF and CO CM in the two channels. Specifically, in the inset of Fig. 4(a) we report the changes in the $B_{1 g}$ and $B_{2 g}$ Ra- man absorption due to a progressive reduction of the CM mass, keeping $T$ and QP-CM coupling fixed. This situation is representative of experiments comparing overdoped samples (large CM mass) with optimally and underdoped samples (with progressively smaller CM mass). The main effect is a shift of weight from low to high $\omega$. The same shift occurs upon reducing both $T$ and CM masses, as can be seen in the main panel of Fig. 4(a), representative of the quantum critical regime for the CM. Notice that, according to the standard expression of Raman vertices [16], in this figure the intensity of the $B_{2 g}$ spectra has been rescaled by a factor $t^{\prime} / t \sim$ 0.2 , where $t^{\prime}$ and $t$ are the next nearest neighbor and the nearest neighbor hopping parameters of a tight-binding model, respectively. Moreover, while the $B_{2 g}$ channel never acquires leading AL corrections from $\mathrm{AF}$ or $\mathrm{CO}$ diagrams (see Table 1), the $B_{1 g}$ absorption may be enhanced if CO CM become increasingly critical when the doping is reduced. In this case, their additional AL contribution appears at frequencies $\omega \lesssim \bar{\Omega} \sim 200 \ldots 300 \mathrm{~cm}^{-1}$. In Fig. 4(b) we report the $B_{1 g}$ absorption arising from the superposition of AF CM acting via $\mathrm{S}+\mathrm{V}$ processes (resummed within a memory-function scheme to remove their singular behavior at low frequency) and $\mathrm{CO}$ CM in the AL channel. Notice that here CO and AF CM have the same mass to schematize their enslaved quantum critical behavior.

Several of the above features are indeed generically observed in LSCO and $\mathrm{Bi}_{2} \mathrm{SrCa}_{2} \mathrm{Cu}_{2} \mathrm{O}_{8}$ (BSCCO) [20]. In the $B_{1 g}$ channel, upon going from overdoped to underdoped systems, weight is shifted to higher frequencies. According to our scheme, this effect could be attributed to AF CM acting via $\mathrm{S}+\mathrm{V}$ processes, and becomes progressively more important upon underdoping, both in LSCO and BSCCO. The effect of $\mathrm{CO} C M$ via $\mathrm{S}+\mathrm{V}$ processes is nearly cancelled in the $B_{1 g}$ channel and the anomalous absorption, which we interpret as due to the $\mathrm{AL}$ contribution, is rather weak even around optimal doping. Upon entering the underdoped region, the marked tendency of LSCO to form stripes makes the $\mathrm{AL}$ contribution of $\mathrm{CO}$ to the $B_{1 g}$ channel more important at low frequencies $\left(\omega \lesssim 200 \mathrm{~cm}^{-1}\right)$. Then, while the $B_{1 g}$ absorption stays moderately suppressed by the AF $\mathrm{S}+\mathrm{V}$ contributions at intermediate frequencies, it acquires a nearly critical additional contribution from the AL processes [15] and displays the anomalous low-frequency absorption shown in Fig. 4(b). Notice that a similar additional anomalous absorption is absent in BSCCO 

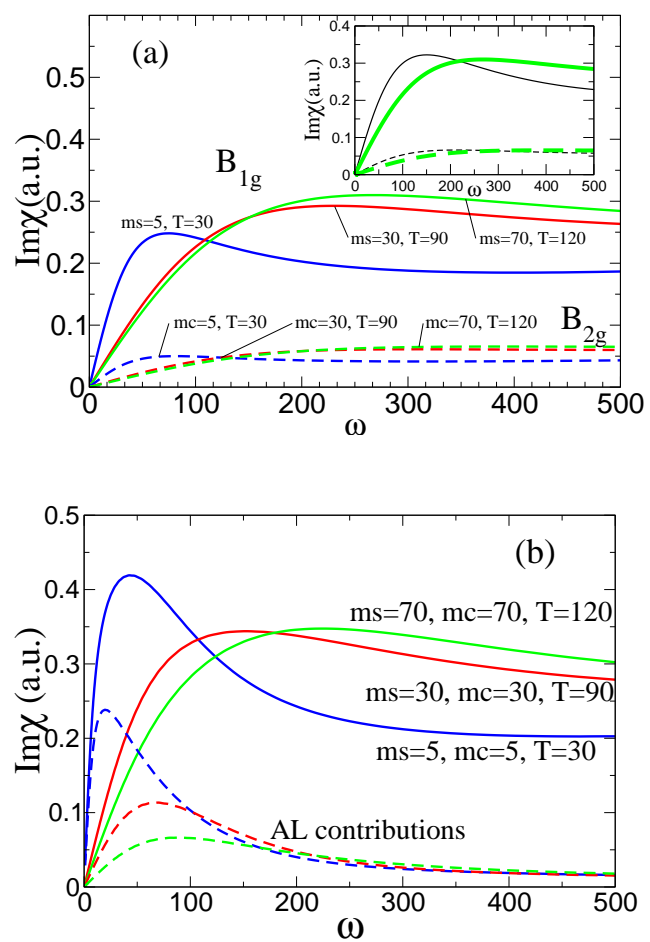

Fig. 4. (a) Raman absorption for a clean system of QP due to $\mathrm{S}+\mathrm{V}$ processes. Solid lines: $B_{1 g}$ channel with QP coupled to AF CM with $\bar{\Omega}_{A F}=500 \mathrm{~cm}^{-1}$; dashed line: $B_{2 g}$ channel with QP coupled to CO CM with $\bar{\Omega}=200 \mathrm{~cm}^{-1}$. $\mathrm{AF}$ and $\mathrm{CO} \mathrm{CM}$ have masses $m s$ and $m c$, respectively. Inset: Same as in the main panel, but with fixed $T=120 \mathrm{~cm}^{-1}$ and two different masses of the CM: $m=70 \mathrm{~cm}^{-1}$ (thick lines) and $m=200 \mathrm{~cm}^{-1}$ (thin lines). (b) Total $B_{1 g}$ Raman absorpion due to $\mathrm{AF} \mathrm{CM}(\mathrm{S}+\mathrm{V}$ diagrams) and to $\mathrm{CO} \mathrm{CM}$ (AL diagrams, also shown separately as dashed lines). Masses and temperatures are the same as in panel (a).

samples. We argue that in BSCCO samples the tendency to form nearly static stripes is much weaker, so that the low-frequency AL absorption could be masked by other processes competing with $\mathrm{CO}$ (e.g., pairing without superconducting phase coherence). Another possibility (not necessarily exclusive) is that in BSCCO the $\mathrm{CO}$ texture has the different form of a checkerboard (previously named "eggbox" in the literature [21]). In this case, symmetry arguments can be found showing that checkerboard charge fluctuations should couple much less to the QP loop entering the AL diagrams of Fig. 1 even for the $B_{1 g}$ symmetry. Of course these non-trivial signatures naturally require more specific parameter tuning and more realistic model calculations (e.g., with specific band structures) and treatment of the cutoffs to improve the quantitative agreement with the behavior of various samples.

In conclusion, the robust symmetry arguments outlined above account for the generic behavior of the Raman spectra at different dopings and in different channels and, contrary to the optical conductivity, allow to distinguish the effects of charge and spin modes.

Acknowledgments We acknowledge interesting discussions with C. Castellani, T. Devereaux, R. Hackl, and J. Lorenzana and financial support from the MIUR-PRIN 2005 - prot. 2005022492 and (CDC and TE) from the Alexander von Humboldt foundation.

\section{References}

[1] C. Castellani, C. Di Castro, and M. Grilli, J. of Phys. and Chem. of Solids 59, 1694 (1998).

[2] C. M. Varma, Phys. Rev. Lett. 75, 898 (1995); Phys. Rev. B. 55, 14554 (1997), and references therein.

[3] J. L. Tallon, J. W. Loram, Physica C 349, 53 (2001).

[4] C. Castellani, C. Di Castro, and M. Grilli, Phys. Rev. Lett. 75, 4650 (1995).

[5] W. Metzner, D. Rohe, and S. Andergassen, Phys. Rev. Lett. 91, 066402 (2003).

[6] S. A. Kivelson, et al., Rev. Mod. Phys. 75, 1201 (2003).

[7] A. Abanov, et al., Adv. Phys. 52, 119 (2003), and references therein.

[8] S. Caprara, et al., Phys. Rev. B 59, 14980 (1999).

[9] G. Seibold and M. Grilli, Phys. Rev. B 63, 224505 (2001).

[10] G. Seibold and M. Grilli, Phys. Rev. B 72, 104519 (2005).

[11] S. Andergassen, et al., Phys. Rev. Lett. 87, 056401 (2001).

[12] S. Caprara, M. Grilli, C. Di Castro, and T. Enss, Phys. Rev. B 75, 140505R (2007).

[13] W. Götze and P. Wölfle, Phys. Rev. B 6, 1226 (1972).

[14] T. Enss, S. Caprara, C. Di Castro, and M. Grilli, in preparation.

[15] S. Caprara, C. Di Castro, M. Grilli, and D. Suppa, Phys. Rev. Lett. 95, 117004 (2005).

[16] See, e.g., T. P. Devereaux, A. Virosztek, and A. Zawadowski, Phys. Rev. B 51, 505 (1995), and references therein.

[17] W. Metzner, C. Castellani, and C. Di Castro, Adv. Phys. 47, 317 (1998)

[18] L. Tassini, et al., Phys. Rev. Lett. 95, 117002 (2005).

[19] F. Venturini, et al., Phys. Rev. B 66, 060502(R) (2002).

[20] R. Hackl, private communication.

[21] G. Seibold, et al., Eur. Phys. J. B 13, 87 (2000). 